\title{
Persistent Descending Mesocolon as a Key Risk Factor in Laparoscopic Colorectal Cancer Surgery
}

\author{
LIMING WANG, HIROKA KONDO, YASUMITSU HIRANO, TOSHIMASA ISHII, KIYOKA HARA, \\ NAO OBARA, MASAHIRO ASARI, TAKUYA KATO, GREGORY HENG and SHIGEKI YAMAGUCHI
}

Division of Gastroenterological Surgery, Saitama Medical University International Medical Center, Hidaka, Japan

\begin{abstract}
Background: Persistent descending mesocolon $(P D M)$ is a rare colonic anatomical variant. However, PDM's impact on the technical aspects and outcomes of laparoscopic colorectal cancer resection are unclear. Patients and Methods: This retrospective clinical cohort study was conducted at a high-volume cancer center in Japan to evaluate intra- and postoperative outcomes of laparoscopic colorectal cancer surgery in patients with (PDM+) or without (PDM-) PDM over the past 7 years. Results: Between January 2012 and September 2019, 2,775 patients underwent laparoscopic colorectal cancer resection at our center, including 60 (2.1\%) cases of PDM. Preoperative detection was achieved in only 5 patients (8.3\%), 39 patients were men, and 21 patients were women. The average age was 67 years. Twenty patients had a history of prior abdominal surgery (33.3\%), with little or no subsequent adhesions. The average duration of sigmoidectomy in PDM+ patients ( $n=17 ; 217.7 \pm 14.2 \mathrm{~min}$ ) was significantly longer than that in PDM- patients ( $n=547 ; 176.2 \pm 2.4 \mathrm{~min}$; $p=0.003)$, as was average blood loss $(32.3 \pm 10.6 \mathrm{ml}$ vs. $16.7 \pm 2.8 \mathrm{ml} ; p=0.03)$. Likewise, average operative time for high anterior resection in PDM+ patients $(n=11 ; 227.1 \pm 20.2$ min) was significantly longer than that in PDM- patients ( $n=294 ; 195.6 \pm 3.0 \mathrm{~min} ; p=0.048)$. Rates of postoperative anastomotic leakage and postoperative recurrence did not differ in both groups. In PDM+ patients, retention of left colic artery had no impact on proximal specimen margins or occurrences of anastomotic leakage. Conclusion: PDM prolongs operative times and increases bleeding in laparoscopic colorectal cancer surgery and should be considered a risk factor when encountered.
\end{abstract}

This article is freely accessible online.

Correspondence to: Liming Wang, MD, Ph.D., Division of Gastroenterological Surgery, Saitama Medical University International Medical Center, Hidaka, Saitama, Japan. Tel: +81 429844111, Fax: +81 429840054, e-mail: wuminami@hotmail.com

Key Words: Persistent descending mesocolon, bear-claw IMA.
Persistent descending mesocolon (PDM) is a rare anatomic variant, characterized by failure of the colonic mesentery of the descending colon to fuse with the abdominal wall (1). Such abnormalities have been documented in radiologic and gynecologic reports since the 1960s $(2,3)$. However, recent publications have detailed specific clinical complications of PDM, such as primary colonic varices, intestinal obstruction, and postoperative anastomotic stenosis (4-7). Since most patients with PDM are asymptomatic before surgery, surgeons are rarely able to identify PDM intraoperative.

The purpose of this retrospective clinical cohort study was to address the intraoperative anatomic characteristics and postoperative outcomes of PDM in the setting of laparoscopic colorectal cancer surgery, and compare patients with and without this anomaly treated at a high-volume cancer center in Japan over the past 7 years.

\section{Patients and Methods}

This review was confined to those patients $(n=2,775)$ who underwent laparoscopic colectomy for primary colorectal cancer between January 2012 and September 2019 at the International Medical Center of Saitama Medical University. Preoperative diagnoses of PDM were established by imaging evidence of rightsided sigmoid and descending colonic shift. During operations, descending mesenteries barely adhered dorsally to retroperitoneum and were inwardly attached to small bowel mesentery or right pelvic wall. Patients with aberrant fixation attributes of PDM determined by prior open surgery were also included if no appreciable adhesions resulted (Figure 1A and B).

Right-sided positioning was used during all left colectomies. Abdominal adhesions were first assessed laparoscopically, and the small intestines were moved cephalad. Adhesions of sigmoid colon to small intestinal or colonic mesentery were typical of PDM. In the event of extensive cecal and right pelvic wall adhesions, operators relocated to the left of patients, freeing ileocecal areas until sigmoid and small bowel mesenteries were completely separated.

Patients positive and negative for PDM were further stratified by laparoscopic procedure performed, namely sigmoidectomy (Lap-S), high anterior resection (Lap-HAR), or low anterior resection (LapLAR), for purposes of comparison. The PDM+ and PDM- groups were assessed in terms of operative time, intraoperative bleeding, intestinal specimen resection, postoperative anastomotic leakage, 
Table I. Baseline characteristics of patients with PDM.

\begin{tabular}{lc}
\hline Gender & 60 \\
Male & $39(65.00)$ \\
Female & $21(35.00)$ \\
Age, years & $67.0 \pm 10.7$ \\
Preoperative diagnosis & $5(8.3)$ \\
Laparoscopic surgery & \\
Sigmoidectomy & $17(28.33)$ \\
High anterior resection & $11(18.33)$ \\
Low anterior resection & $17(28.33)$ \\
Descending colectomy & $3(5.00)$ \\
Intersphincteric resection & $6(10.00)$ \\
Hartmann procedure & $2(3.33)$ \\
Abdominoperineal resection & $2(3.33)$ \\
Ileocecectomy & $2(3.33)$ \\
Prior surgery & $20(33.33)$ \\
Appendectomy & $14(70.00)$ \\
Nephrectomy & $2(10.0)$ \\
Laparoscopic hernia repair & $1(5.00)$ \\
Cholecystectomy & $2(10.00)$ \\
Ovariectomy & $1(5.00)$ \\
Complication & $11(18.33)$ \\
Bowel obstruction & $1(1.67)$ \\
Anastomotic leakage & $5(8.33)$ \\
Stomal hernia & $1(1.67)$ \\
Early postoperative hernia & $1(1.67)$ \\
Right peroneal nerve paralysis & $1(1.67)$ \\
Lymphatic leakage & $1(1.67)$ \\
Dysuria & $1(1.67)$ \\
LCA retention & $36(60)$ \\
Bear-claw IMA configuration & $4 / 36(11.11)$ \\
\hline
\end{tabular}

Data expressed as $\mathrm{n}, \mathrm{n}(\%)$, or mean \pm SD. PDM, Persistent descending mesocolon; LCA, left colic artery; IMA, inferior mesenteric artery; SD, standard deviation.

and postoperative recurrence, separately analyzing anastomotic leakages, operative time, and bleeding in those with preserved and non-preserved left colic arteries.

All statistical analyses were powered by standard software (SPSS v22 for Mac; IBM Corp, Armonk, NY, USA). Chi-square and Fisher's exact tests for categorical variables were applied to ascertain differences, setting significance at $p<0.05$.

\section{Results}

There were $60(2.1 \%)$ patients with PDM, comprising of 39 men and 21 women. Average age was 67 years, and five $\mathrm{PDM}+$ patients $(8.3 \%)$ were diagnosed by preoperative computed tomography $(\mathrm{CT})$. Prevailing surgical procedures (Table I) were distributed as follows: Lap-S, 17 patients (28.3\%); Lap-HAR, 11 patients (18.3\%); and Lap-LAR, 17 patients $(28.3 \%)$. Two patients underwent ileocecal resections. In 20 patients (33.3\%) with history of abdominal operations (14 cases were appendectomies), no surgically induced adhesions were present to obscure fixation defects of PDM. Eleven patients (18.3\%) experienced postoperative complications, including five anastomotic leakages $(8.3 \%)$ and three occurrences (one each) of intestinal obstruction, lymphatic leak, and hernia.

Left colic artery (LCA) was preserved in 36 patients, four of whom showed same-level inferior mesenteric artery (IMA) branching of LCA, superior rectal artery (SRA), and sigmoid arteries (SAs) in a pattern resembling bear claws (referred to as "bear-claw IMA") (Figure 2A). Under such circumstances, the left colic artery is usually much shorter and may directly give rise to the marginal artery (Figure $2 \mathrm{~B}$ and $\mathrm{C}$ ).

Next, we compared patient groups with and without PDM after Lap-S. The average duration of Lap-S in 17 PDM+ patients $(217.7 \pm 14.2 \mathrm{~min})$ significantly exceeded that in 547 PDM- patients $(176.2 \pm 2.4 ; p=0.003)$, as did blood loss $(32.3 \pm 10.6 \mathrm{ml} v s .16 .7 \pm 2.8 \mathrm{ml} ; p=0.03)$. Postoperative feeding resumed in a median of 3 days, and median hospital stay was 7.0 days, neither differing significantly by group. Proportions of double-stapling technique (DST) and functional end-to-end anastomosis (FEEA) were similar in the two groups, and there was no significant difference in the lengths of proximal $(9.9 \pm 0.29 \mathrm{~cm}$ vs. $10.1 \pm 0.1 \mathrm{~cm})$ or distal $(8.7 \pm 1.06 \mathrm{~cm} v s$. $8.5 \pm 0.13 \mathrm{~cm})$ resection margins or in respective rates of anastomotic leakage (5.8\% vs. $1.2 \% ; p=0.114)$. Recurrences developed in three patients of the PDM+ group. Although higher than that in the PDM group ( $17.6 \%$ vs. $8.5 \%$; $p=0.195)$, the difference was not significant (Table II).

We subsequently analyzed 11 PDM+ and 294 PDMpatients undergoing Lap-HAR. Mean age and male-to-female ratio did not differ significantly by group. Mean operative time was significantly longer in the PDM+ group $(227.1 \pm 20.2 \mathrm{~min} v s$. $195.6 \pm 3.0 \mathrm{~min} ; p=0.048)$, but the difference in blood loss totals was marginal $(25.7 \pm 7.5 \mathrm{ml} v s$. $20.9 \pm 1.6 \mathrm{ml} ; p=0.643)$. Resumption times for postoperative feeding and hospital stays were similar, and there was no significant difference in rates of postoperative anastomotic leakage $(9.0 \%$ vs. $2.3 \% ; p=0.375)$ or disease recurrence (18.1\% vs. 9.5\%; $p=0.343)$ (Table III).

In the PDM+ (vs. PDM-) group, operative times for LapLAR were longer $(266.0 \pm 12.6 \mathrm{~min} v s .252 \pm 3.2 \mathrm{~min} ; p=0.457)$, and greater blood loss was evident $(32.2 \pm 14.3 \mathrm{ml}$ vs. $24.6 \pm 1.9$ $\mathrm{ml} ; p=0.532$ ), but statistical significance was not reached. The groups were also similar in terms of background, intraoperative bleeding, postoperative recovery, proximal resection margin $(13.5 \pm 1.07 \mathrm{~cm}$ vs. $13.7 \pm 1.08 \mathrm{~cm})$, anastomotic leakage $(9.43 \%$ vs. $11.76 \% ; p=0.746)$, and postoperative recurrence $(13.5 \%$ vs. $5.8 \% ; p=0.360$ ) (Table IV).

As a final effort, we stratified PDM+ patients by LCA status, comparing those with $(n=36)$ or without $(n=22)$ LCA preservation. Patients undergoing abdominoperineal resections, Hartmann procedures, and functional end-to-end anastomoses (FEEAs, n=4) were excluded, leaving 18 LCA+ and 17 LCA-patients, respectively for analysis. However, these groups did not differ significantly in terms of 

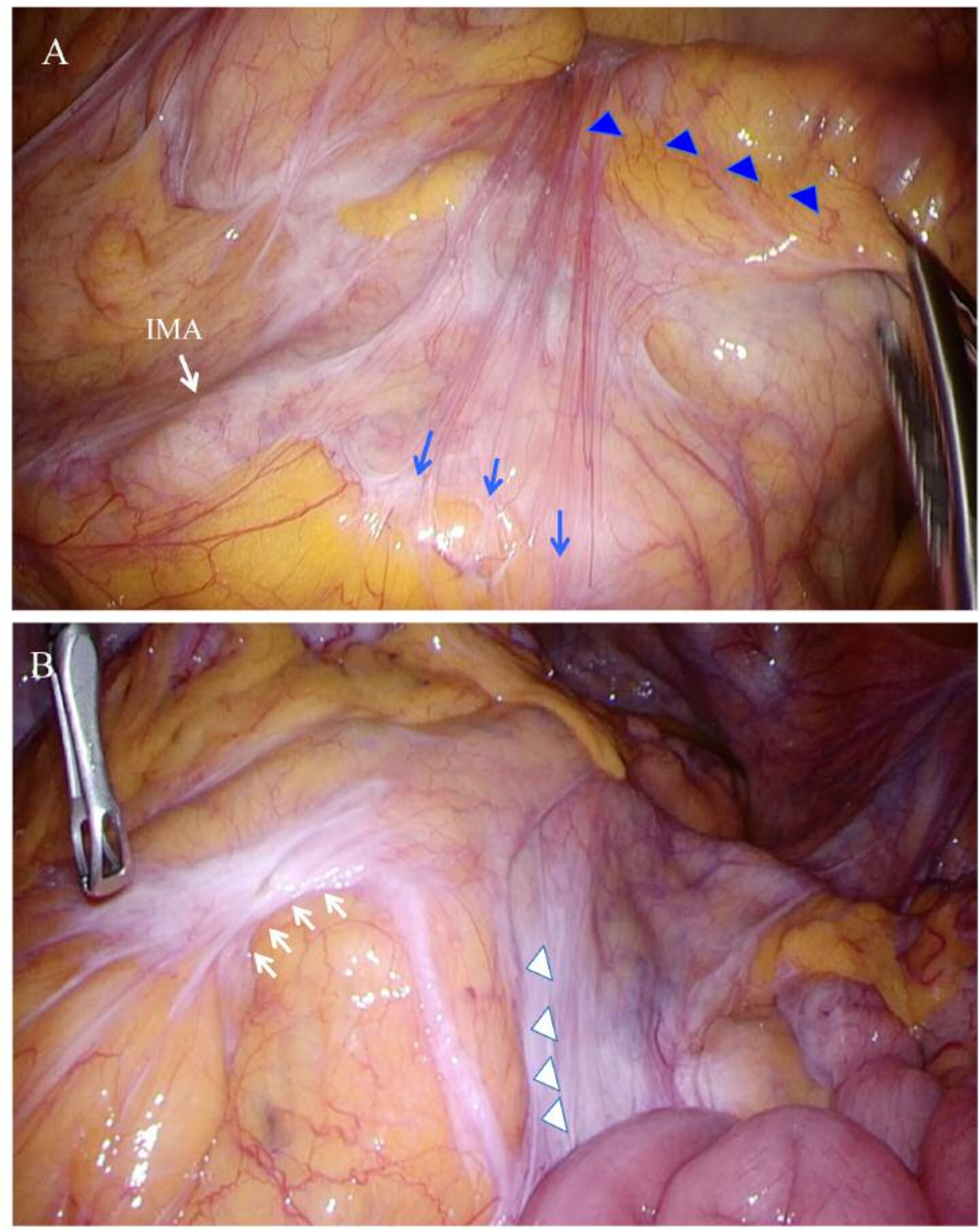

Figure 1. Intraoperative classification of persistent descending mesocolon (PDM). (A) Simple PDM: adherent mesenteries of descending colon and small intestine (blue arrows), descending colon marked by blue arrowheads; medial approach to freeing inferior mesenteric artery (IMA) unaffected. (B) Complex PDM: self-adhesion of descending colonic mesentery (white arrows) and adherence to mesentery of right small intestine (white arrow heads); medial approach to freeing buried IMA extremely difficult.

background, operative method, operative time $(258.1 \pm 17.5$ $\min v s .236 \pm 14.9 \mathrm{~min})$, blood loss $(33.2 \pm 14.0 \mathrm{ml}$ vs. $15.3 \pm 3.1 \mathrm{ml})$, or length of pathologic proximal margin $(13.24 \pm 1.02 \mathrm{~cm} \mathrm{vs.} 12.89 \pm 1.09 \mathrm{~cm})$; and there were two patients in each group with anastomotic leakages (Table V).

$\mathrm{PDM}+$ and PDM- patients did not differ significantly in terms of the American Society of Anesthesiologists (ASA) classification system, preoperative tumor diameters and infiltrative depths.

\section{Discussion}

In patients with PDM, the descending colon may assume a more intermediate position than usual, with a shift in sigmoid colon to right side of abdomen. PDM is, thus, referred to at times as "right-sided sigmoid colon" (8). Anomalous internal fixation of the fetal mesentery may cause colonic dislocation, but the left (rather than right) colon is less apt to be affected than the right (9). At $\sim 5$ months of gestation, mesenteries of 


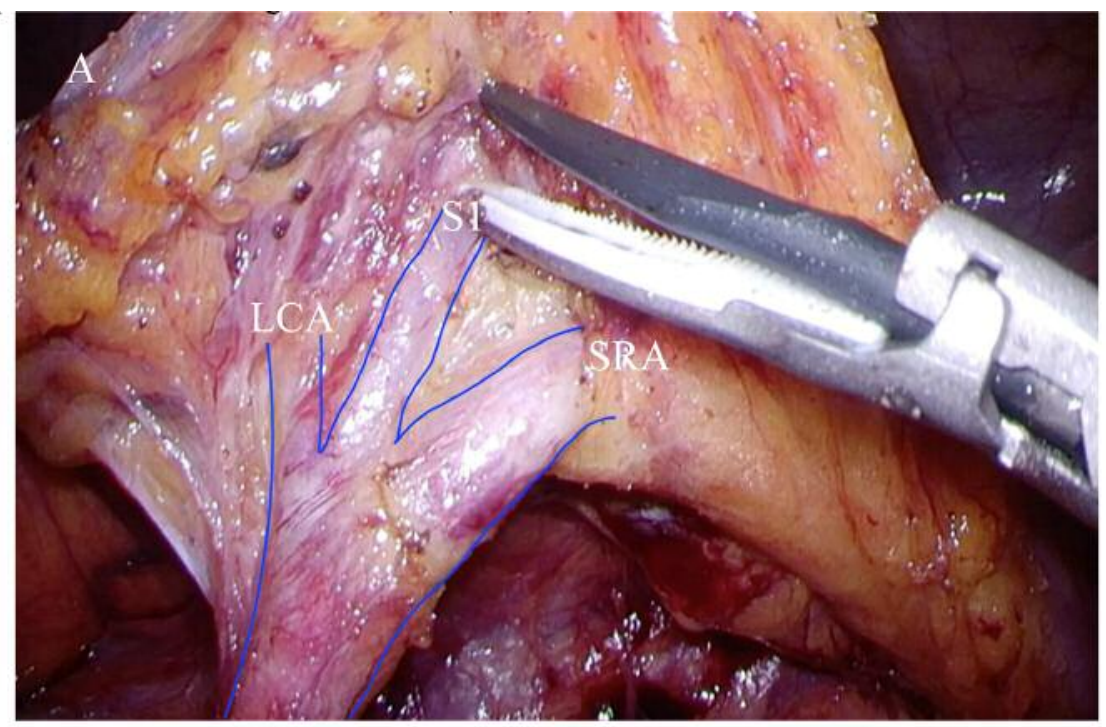

B

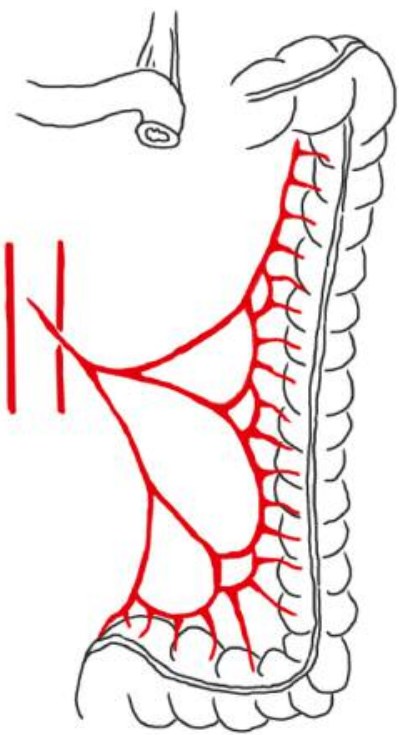

IMA in no-PDM

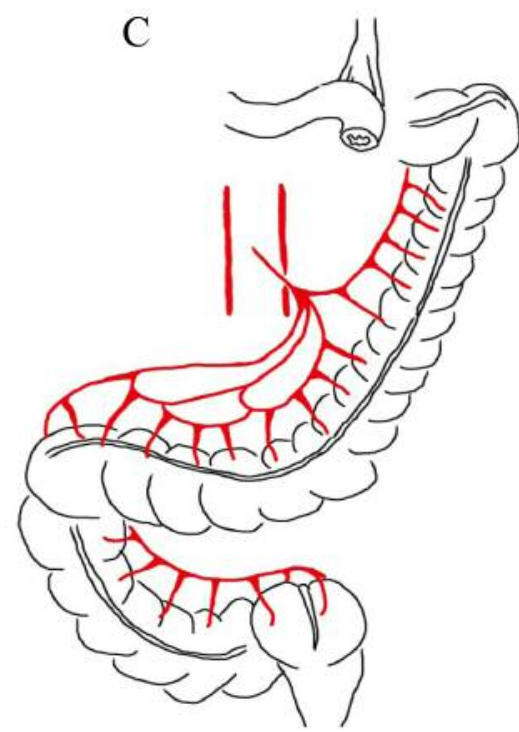

Bear-claw IMA in PDM

Figure 2. Bear-claw inferior mesenteric artery (IMA) configuration typical of persistent descending mesocolon (PDM). (A) IMA branching at same level [left colic artery (LCA), first or second sigmoid artery (S1 or S2), and superior rectal artery (SRA)] creating characteristic three-pronged bear claw. (B) Anatomy of normal IMA in absence of PDM. (C) Bear-claw IMA of PDM associated with medial shift of descending colon and potential left colic arterial origin of marginal artery.

both ascending and descending colon generally fuse with the peritoneal wall, becoming permanently fixed to retroperitoneum. In PDM, imaging studies typically show medial movement of descending colon due to aberrant mesenteric fixation, thereby freeing the left iliac fossa and left outer abdominal wall. The small intestine may then occupy this space $(1,3)$. Nevertheless, we identified only five cases (8.3\%) of PDM by preoperative CT imaging, so a fundamental awareness of PDM anatomy is particularly important for intraoperative recognition.

Patients with PDM customarily show adhesions and shortening of the descending colonic mesentery. In some cases, the descending mesentery is self-adherent or attached to mesentery of transverse colon, and most develop adhesions to small bowel mesentery. Excessive adhesions in $\mathrm{PDM}+$ patients have been reported to interfere with right 
Table II. Lap-S group demographics and operative characteristics.

\begin{tabular}{lccc}
\hline Characteristics & PDM+ & PDM- & $p$-Value \\
\hline Gender & 17 & 547 & \\
$\quad$ Male & $12(70.59)$ & $332(60.69)$ & \\
Female & $5(29.41)$ & $215(39.31)$ & NS \\
Age, years & $71.1 \pm 1.8$ & $66.3 \pm 0.46$ & NS \\
Operative time, min & $217.7 \pm 14.2$ & $176.2 \pm 2.4$ & 0.003 \\
Operative blood loss, ml & $32.3 \pm 10.6$ & $16.7 \pm 2.8$ & 0.03 \\
Food intake, POD & & & \\
$\quad$ Median & $3(3-17)$ & $3(2-22)$ & \\
$\quad$ Mean & $4.1 \pm 0.84$ & $3.2 \pm 0.04$ & NS \\
Discharge, POD & & & \\
$\quad$ Median & $7(6-90)$ & $7(4-67)$ & \\
$\quad$ Mean & $11.4 \pm 1.2$ & $7.4 \pm 0.26$ & NS \\
Anastomotic method & $13(76.47)$ & $368(67.28)$ & \\
$\quad$ DST & $4(23.53)$ & $179(32.72)$ & NS \\
$\quad$ FEEA & & & \\
Resection margin, cm & $9.9 \pm 0.29$ & $10.1 \pm 0.1$ & NS \\
$\quad$ Proximal & $8.7 \pm 1.064$ & $8.5 \pm 0.13$ & NS \\
$\quad$ Distal & $1(5.88)$ & $7(1.28)$ & 0.114 \\
Anastomotic leakage & $3(17.65)$ & $47(8.59)$ & 0.195 \\
Recurrence &
\end{tabular}

Data expressed as $\mathrm{n}, \mathrm{n}(\%)$, median (range), or mean \pm SD. PDM, Persistent descending mesocolon; Lap-S, laparoscopic sigmoidectomy; POD, postoperative day; SD, standard deviation; DST, double-stapling technique; FEEA, functional end-to-end anastomosis; NS, not significant.

Table IV. Lap-LAR group demographics and operative characteristics.

\begin{tabular}{lccc}
\hline Characteristics & PDM+ & PDM- & $p$-Value \\
\hline Gender & 17 & 562 & \\
$\quad$ Male & $13(65.66)$ & $369(76.47)$ & \\
$\quad$ Female & $4(34.34)$ & $193(23.53)$ & NS \\
Age, years & $62.6 \pm 2.9$ & $64.1 \pm 0.35$ & NS \\
Operative time, min & $266.0 \pm 12.6$ & $252 \pm 3.2$ & 0.457 \\
Operative blood loss, ml & $32.2 \pm 14.3$ & $24.6 \pm 1.9$ & 0.532 \\
Food intake, POD & & & \\
$\quad$ Median & $3(3-6)$ & $3(2-53)$ & \\
$\quad$ Mean & $3.3 \pm 0.2$ & $4.06 \pm 0.16$ & NS \\
Discharge, POD & & & \\
$\quad$ Median & $9(6-36)$ & $9(5-136)$ & \\
$\quad$ Mean & $11 \pm 1.7$ & $12.29 \pm 0.53$ & NS \\
Resection margin, cm. & & & \\
$\quad$ Proximal & $13.5 \pm 1.07$ & $13.7 \pm 1.08$ & \\
$\quad$ Distal & $3.1 \pm 0.33$ & $2.9 \pm 0.41$ & NS \\
Anastomotic leakage & $2(9.43)$ & $53(11.76)$ & 0.746 \\
Recurrence & $1(13.52)$ & $76(5.88)$ & 0.360 \\
\hline
\end{tabular}

Data expressed as n, n (\%), median (range), or mean \pm SD. PDM, Persistent descending mesocolon; Lap-LAR, laparoscopic low anterior resection; $\mathrm{POD}$, postoperative day; SD, standard deviation; NS, not significant.

hemicolectomy (10). In two of our patients with PDM, intraoperative relocation of the surgeon was required to remove adhesions during right colon resection.
Table III. Lap-HAR group demographics and operative characteristics.

\begin{tabular}{lccc}
\hline Characteristics & PDM+ & PDM- & $p$-Value \\
\hline Gender & 11 & 294 & \\
Male & $7(63.64)$ & $178(60.54)$ & \\
Female & $4(36.36)$ & $116(39.46)$ & $\mathrm{NS}$ \\
Age, years & $71.2 \pm 2.1$ & $67.1 \pm 0.69$ & $\mathrm{NS}$ \\
Operative time, min & $227.1 \pm 20.2$ & $195.6 \pm 3.0$ & 0.048 \\
Operative blood loss, ml & $25.7 \pm 7.5$ & $20.9 \pm 1.6$ & 0.643 \\
Food intake, POD & & & \\
$\quad$ Median & $3(3-30)$ & $3(2-45)$ & \\
$\quad$ Mean & $5.8 \pm 2.42$ & $3.4 \pm 0.17$ & $\mathrm{NS}$ \\
Discharge, POD & & & \\
$\quad$ Median & $6(6-35)$ & $7(5-94)$ & \\
$\quad$ Mean & $11.45 \pm 2.87$ & $7.89 \pm 0.42$ & $\mathrm{NS}$ \\
Resection margin, cm & & & \\
$\quad$ Proximal & $12.64 \pm 1.01$ & $11.9 \pm 0.18$ & $\mathrm{NS}$ \\
$\quad$ Distal & $4.36 \pm 0.39$ & $4.7 \pm 0.1$ & $\mathrm{NS}$ \\
Anastomotic leakage & $1(9.09)$ & $7(2.38)$ & 0.375 \\
Recurrence & $2(18.18)$ & $28(9.52)$ & 0.343 \\
\hline
\end{tabular}

Data expressed as $\mathrm{n}, \mathrm{n}(\%)$, median (range), or mean \pm SD. PDM, Persistent descending mesocolon; Lap-HAR, laparoscopic high anterior resection; POD, postoperative day; SD, standard deviation; NS, not significant.

Table V. Demographic and operative characteristics of PDM+ group by LCA status.

\begin{tabular}{lccc}
\hline Characteristics & LCA- & LCA+ & $p$-Value \\
\hline Gender & 17 & 18 & \\
$\quad$ Male & $10(58.82)$ & $15(83.33)$ & \\
$\quad$ Female & $7(41.18)$ & $3(16.67)$ & 0.109 \\
Age, years & $67.1 \pm 2.4$ & $68.2 \pm 2.1$ & NS \\
Laparoscopic surgery & & & \\
$\quad$ Sigmoidectomy (DST) & $4(23.53)$ & $5(27.78)$ & \\
$\quad$ High anterior resection & $4(23.53)$ & $6(33.33)$ & \\
$\quad$ Low anterior resection & $7(41.18)$ & $5(27.78)$ & \\
$\quad$ Intersphincteric resection & $2(11.76)$ & $2(11.11)$ & NS \\
Operative time, min & $258.1 \pm 17.5$ & $236 \pm 14.9$ & $\mathrm{NS}$ \\
Operative blood loss, ml & $33.2 \pm 14.0$ & $15.3 \pm 3.1$ & $\mathrm{NS}$ \\
Resection margin, cm & & & \\
$\quad$ Proximal & $13.24 \pm 1.02$ & $12.89 \pm 1.09$ & $\mathrm{NS}$ \\
Anastomotic leakage & $2(11.76)$ & $2(11.11)$ & 0.951 \\
\hline
\end{tabular}

Data expressed as n, n (\%), or mean \pm SD. LCA: left colic artery; PDM, persistent descending mesocolon; SD, standard deviation; NS, not significant; DST, double-stapling technique.

According to surgical impact, PDM is categorized as simple or complex. Adhesions are locally confined in simple PDM, so medial dissection may proceed unhindered, easily locating IMA for lymph node dissection (Figure 1A). In complex PDM, the process of separating extensive adhesions to identify IMA may be time-consuming and challenging, increasing the chance of intraoperative bleeding (Figure 1B). This accounts for the significantly longer operative times and the greater blood loss in PDM+ patients undergoing laparoscopic sigmoidectomy. 
Shortening of the descending colonic mesentery is another hallmark of PDM leading to possible vascular variations. Still, when comparing postoperative outcomes in PDM patient subsets with or without LCA preservation, we found no significant difference in operative time, intraoperative blood loss, proximal and distal lengths of intestinal specimen, or postoperative anastomotic leakage. Additionally, there were four $(11.1 \%)$ patients with classic bear-claw IMA configurations among the $36 \mathrm{PDM}+$ patients with intact LCAs (Figure 2A and C), one in particular showing direct LCA origin for the marginal artery supplying intestinal canal. Once the LCA was ligated, considerable intestinal ischemia ensued, necessitating removal of a nearly $50-\mathrm{cm}$ segment. Especially in the presence of extensive adhesions, surgeons must be mindful of the risks inherent in bear-claw variants of IMA, although it is still controversial whether LCA is critical in sigmoidal and rectal surgeries $(11,12)$. LCA ligation does not significantly increase the risk of postoperative anastomotic leakage in PDM+ patients. In those with bear-claw IMAs, where marginal artery may originate from LCA, indocyanine green (ICG) imaging is perhaps indicated to better ascertain intestinal blood flow $(4,13,14)$.

The detection of PDM by preoperative imaging is limited, hence the above three features (i.e., medial shift of descending colon, shortening of descending mesentery, and bear-claw IMA vascular pattern) should alert the surgeon to the presence of PDM in the operative setting. Once confirmed, the surgical focus should be on proper isolation of layers and control of potential bleeding caused by anomalous vascularity.

We found it curious that operative times and blood losses during Lap-LAR did not differ significantly in PDM+ and PDM- groups. A likely reason is the fact that such comparatively lengthy procedures tend to marginalize statistical differences. Another explanation resides in the degree of surgical complexity, which is beyond that of Lap-S and LapHAR. A senior physician in our hospital performed most of the Lap-LAR operations. For doctors skilled in laparoscopic surgery, PDM is not inordinately problematic, but young surgeons must quickly acquire the expertise needed in this field, climbing the learning curve in short order. Admittedly, the number of PDM+ patients treated at our center was rather small, and statistical bias imposed by operator factors may be corrected via propensity score matching. We look forward to a more objective understanding of PDM through multicenter prospective clinical trials.

\section{Conclusion}

PDM prolongs operative times and increases bleeding in laparoscopic colorectal cancer surgery and should be considered a risk factor when encountered.

\section{Consent for Publication}

All patients have agreed to use their personal medical data for research and publication.

\section{Ethics Approval and Consent to Participate}

All study participants provided their informed consent. The Ethics Committee of the Saitama Medical University International Medical Center approved the study.

\section{Conflicts of Interest}

The Authors have no competing interests to declare with respect to this study.

\section{Authors' Contributions}

LMW and HK drafted the manuscript and provided original pictures. YH, TI, KH, NO, MA, TK, GH and SY reviewed its content. All Authors have read and approved the final submission.

\section{Acknowledgements}

Our thanks are extended to BioMed Proofreading LLC for English copyediting.

\section{References}

1 Popky GL and Lapayowker MS: Persistent descending mesocolon. Radiology 86(2): 327-331, 1966. PMID: 5902472. DOI: $10.1148 / 86.2 .327$

2 Balthazar EJ: Congenital positional anomalies of the colon: Radiographic diagnosis and clinical implications. Ii. Abnormalities of fixation. Gastrointest Radiol 2(1): 49-56, 1977. PMID: 615802. DOI: 10.1007/BF02256465

3 Morgenstern L: Persistent descending mesocolon. Surg Gynecol Obstet 110: 197-202, 1960. PMID: 14424134.

4 Hiyoshi Y, Miyamoto Y, Eto K, Nagai Y, Iwatsuki M, Iwagami $\mathrm{S}$, Baba $\mathrm{Y}$, Yoshida $\mathrm{N}$ and Baba $\mathrm{H}$ : Laparoscopic surgery for colorectal cancer with persistent descending mesocolon. World J Surg Oncol 17(1): 190, 2019. PMID: 31711517. DOI: 10.1186/ s12957-019-1734-1

5 Ongom PA, Lukande RL and Jombwe J: Anal protrusion of an ileo-colic intussusception in an adult with persistent ascending and descending mesocolons: A case report. BMC Res Notes 6: 42, 2013. PMID: 23375100. DOI: 10.1186/1756-0500-6-42

6 Kanai M, Tokunaga T, Miyaji T, Mataki N, Okada C, Mitani K, Aono S, Kobari S and Hakozaki Y: Colonic varices as a result of persistent mesocolon of the ascending and descending colon. Endoscopy 43 Suppl 2 UCTN: E103-104, 2011. PMID: 21424996. DOI: $10.1055 / \mathrm{s}-0030-1256137$

7 Tsuruta A, Watanabe Y, Oka Y, Fujita M, Matsumoto H, Fujiwara Y, Shiotani A and Ueno T: Relevance of a persistent descending mesocolon to sigmoid volvulus. Clin Surg 3: 2188, 2018.

8 Wondrak E: Right-side fixation of the sigmoid colon. Rozhl Chir 36(3): 158-160, 1957. PMID: 13467126. 
9 Shrivastava P, Tuli A, Kaur S and Raheja S: Right sided descending and sigmoid colon: Its embryological basis and clinical implications. Anat Cell Biol 46(4): 299-302, 2013. PMID: 24386604. DOI: 10.5115/acb.2013.46.4.299

10 Tsuruta A, Kawai A, Oka Y, Okumura H, Matsumoto H, Hirai T and Nakamura M: Laparoscopic right hemicolectomy for ascending colon cancer with persistent mesocolon. World J Gastroenterol 20(18): 5557-5560, 2014. PMID: 24833887. DOI: 10.3748/wjg.v20.i18.5557

11 Wang L, Hirano Y, Ishii T, Kondo H, Hara K, Obara N, Tan P and Yamaguchi S: Diverting stoma versus no diversion in laparoscopic low anterior resection: A single-center retrospective study in japan. In Vivo 33(6): 2125-2131, 2019. PMID: 31662547. DOI: 10.21873 /invivo.11713

12 Nayeri M, Iskander O, Tabchouri N, Artus A, Michot N, Muller O, Giger-Pabst U, Bourlier P, Kraemer-Bucur A, Lecomte T, Salame E and Ouaissi M: Low tie compared to high tie vascular ligation of the inferior mesenteric artery in rectal cancer surgery decreases postoperative complications without affecting overall survival. Anticancer Res 39(8): 4363-4370, 2019. PMID: 31366531. DOI: 10.21873 /anticanres.13605
13 Watanabe J, Ishibe A, Suwa Y, Suwa H, Ota M, Kunisaki C and Endo I: Indocyanine green fluorescence imaging to reduce the risk of anastomotic leakage in laparoscopic low anterior resection for rectal cancer: A propensity score-matched cohort study. Surg Endosc, 2019. PMID: 30877565. DOI: 10.1007/ s00464-019-06751-9

14 Ryu S, Suwa K, Kitagawa T, Aizawa M, Ushigome T, Okamoto T, Eto $\mathrm{K}$ and Yanaga K: Real-time fluorescence vessel navigation using indocyanine green during laparoscopic colorectal cancer surgery. Anticancer Res 39(6): 3009-3013, 2019. PMID: 31177142. DOI: 10.21873/anticanres. 13433

Received December 9, 2019 Revised December 16, 2019 Accepted January 5, 2020 